\title{
Extramedullary versus intramedullary femoral alignment technique in total knee arthroplasty: a meta-analysis of randomized controlled trials
}

\author{
Qian Tang ${ }^{1+}$, Ping Shang ${ }^{2+}$, Gang Zheng ${ }^{1}$, Hua-Zi Xu ${ }^{1}$ and Hai-Xiao Liu ${ }^{1 *}$ (D)
}

\begin{abstract}
Background: There is no consensus whether the use of the extramedullary femoral cutting guide takes advantage over the intramedullary one in total knee arthroplasty. The aim of this study was to compare the extramedullary femoral alignment guide system with the conventional intramedullary alignment guide system for lower limb alignment, blood loss, and operative time during total knee arthroplasty.

Methods: The Medline, Embase, Cochrane Library, China National Knowledge Infrastructure (CNKI), Wan Fang Chinese Periodical, Google, and reference lists of all the included studies were searched for randomized controlled trials. The following parameters were compared between the extramedullary technique and the intramedullary technique: (1) lower limb coronal alignment, (2) coronal alignment of femoral component, (3) sagittal alignment of femoral component, (4) blood loss, (5) and operation time.

Results: Four randomized controlled trials consisting of 358 knees were included in our study. There was no significant difference between the extramedullary and intramedullary groups for the lower limb coronal alignment $(\mathrm{RR}=1.20,95 \% \mathrm{Cl} 0.28 \sim 5.21$, n.s.), coronal alignment of femoral component $(\mathrm{RR}=0.65,95 \% \mathrm{Cl} 0.19 \sim 2.22$, n.s.), and sagittal alignment of femoral component $(\mathrm{RR}=0.73,95 \% \mathrm{Cl} 0.38 \sim 1.41$, n.s.). A reduced blood loss was associated with the use of the extramedullary guide $(M D=-120.34,95 \% \mathrm{Cl}-210.08 \sim-30.59, P=0.009)$. No significant difference in operation time was noted between the two groups ( $\mathrm{MD}=1.41,95 \% \mathrm{Cl}-1.82 \sim 4.64$, n.s.).

Conclusions: Neither extramedullary nor intramedullary femoral alignment is more accurate than the other in facilitating the femoral cut in total knee arthroplasty. Use of the extramedullary guide results in less blood loss and exhibits a similar operation time as compared with the intramedullary guide.
\end{abstract}

Keywords: Total knee arthroplasty, Meta-analysis, Blood loss

\section{Background}

In total knee arthroplasty (TKA), the prosthetic placement and overall limb alignment has been demonstrated to be most influential in determining implant survival [1-4]. The ideal position for the components recreates a neutral mechanical axis. Most surgeons currently favor intramedullary (IM) alignment for its ease of use and

\footnotetext{
* Correspondence: spineliu@163.com

${ }^{\dagger}$ Equal contributors

'Department of Orthopaedic Surgery, The Second Affiliated Hospital and Yuying Children's Hospital of Wenzhou Medical University, 109, Xueyuanxi road, 325027 Wenzhou, China

Full list of author information is available at the end of the article
}

accuracy as compared with extramedullary (EM) alignment. Previous comparisons of IM and EM femoral alignment systems have shown the former to be more accurate in performing the distal femoral cut [5-7]. In these studies, the IM alignment technique has ranged from 85 to $96 \%$ in the normal range as compared with 69 to $86 \%$ for the EM alignment technique.

However, the IM femoral alignment system does not always guarantee the accuracy of the component position in TKA. Previous studies used only the anterosuperior iliac spine as an intraoperative landmark for the EM referencing instruments [5-7]. Intraoperative visual 
assessment of the longitudinal femur axis and the anterosuperior iliac spine by EM rods is difficult owing to the large soft tissue cover and tourniquets. TKAs using extramedullary femoral alignment guides have been extensively studied in recent years. Extramedullary instruments using newly designed mechanical axis marker systems have provided as accuracy in reproducing a neutral distal femoral resection on the coronal and sagittal planes during TKA as standard IM instruments [8-10].

Recent advances in TKA have focused on the reduction of damage during the procedure [11-13]. One of the most invasive parts of TKA is the violation of the intramedullary femoral canal and the subsequent use of IM instruments. The use of an intramedullary guide for the femur can result in various complications such as blood loss, postoperative hypoxia, intraoperative fractures, and fat embolism [11, 14, 15], while the EM method has less morbidity in terms of blood loss because it does not invade bone marrow. In addition, the IM instrument may not be applicable when a long-stemmed femoral component implanted during a previous surgery remains or a rod cannot be inserted due to severe deformity of the femur [9].

In this study, we conducted a meta-analysis of pooled the data from relevant RCTs to evaluate whether an IM or EM femoral guide is more accurate in assuring correct femoral positioning. Moreover, the blood loss and operation time were also compared between these two techniques. Our hypothesis was that the EM femoral guide provided similar accuracy for femoral positioning and less blood loss compared with the IM femoral guide during TKAs. If the hypothesis is confirmed, the EM femoral guide may provide an alternative approach for femoral cuts and display clinical benefit for particular cases, such as a previous surgery remains and severe deformity of the femur.

\section{Methods \\ Database retrieval}

The present study was conducted using the Preferred Reporting Items for Systematic Reviews and Meta Analyses (PRISMA) statement. We conducted this metaanalysis of all English and non-English articles identified from electronic databases including Medline, Embase, Cochrane Library, China National Knowledge Infrastructure, Wan Fang Chinese Periodical, and Google. In addition, we also manually searched for other relevant studies including those from the reference lists of all included studies. The last search was conducted on December 6, 2015. We used the following key words: arthroplasty, replacement, femoral, total knee arthroplasty, randomized, randomised, intramedullary, extramedullary, in combination with the Boolean operators AND or OR. The search strategy is presented in Fig. 1.

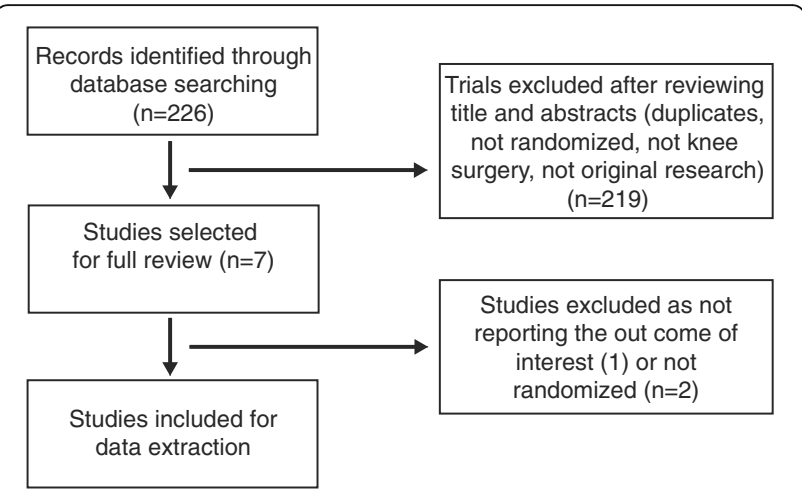

Fig. 1 Flow chart of the study selection and inclusion process

\section{Quality criteria of trials and data extraction}

We included all published RCTs comparing EM guide with IM guide in patients undergoing primary TKA. Exclusion criteria comprised the following: trials with a retrospective design and trials that did not randomize patients into two relevant groups. Quality criteria included the randomization method, concealment of allocation, blinding, and intention-to-treat analysis.

For each eligible study, two of the authors of this metaanalysis independently extracted all relevant data. Disagreement was resolved by discussion with a third investigator. The following data were extracted: (1) the participants' demographic data; (2) the lower limb coronal alignment; (3) the coronal alignment of femoral component; (4) the sagittal alignment of femoral component; (5) the blood loss; (6) and the operation time. When data were incomplete or unclear, attempts were made to contact the investigators for clarification. The lower limb coronal alignment was defined as a line bisecting the center of the femoral head, the center of the knee, and the center of the ankle. The coronal alignment of femoral component (which represents varus-valgus angulation) was measured in the coronal plane on the full-limb anteroposterior film. The sagittal alignment of femoral component (which represents flexion-extension angulation) was measured in the sagittal plane on the lateral film [9].

\section{Statistical methods}

This meta-analysis was conducted using RevMan 5.0 (Cochrane Collaboration, Oxford, UK). We assessed the statistical heterogeneity using a standard chi-square test (statistical heterogeneity was considered to be present at $P<0.1$ and $I^{2}$ values $>50 \%$ ). When comparing trials exhibiting heterogeneity, pooled data were meta-analyzed using a random effects model; otherwise, a fixed effects model was used. Mean differences and 95\% confidence intervals (CIs) were calculated for continuous outcomes and risk ratio (RR) and 95\% CIs for dichotomous outcomes. Ethical approval was obtained. 


\section{Results}

\section{The included studies}

A total of 226 potentially relevant papers were identified. By screening titles and reading the abstracts and entire articles, four studies with 358 knees (178 in the EM group and 180 in the IM group) were included in the final meta-analysis. All of the included studies were RCTs which were level I evidence studies and all published in English. The sample sizes ranged from 50 to 100 knees. The key characteristics of the included RCTs are summarized in Table 1 . And the detailed data of the comparison of blood loss between femoral EM and IM technique was showed in Additional file 1.

\section{Quality of studies}

The methodologic quality of the four included studies was variable. The reported methods of generating allocation sequences were adequate in two studies and one trial reported allocation concealment. Blinding of surgeon and patients were reported in one study and one of the studies blinded their assessors to the outcome. The methodologic quality of the studies is presented in Fig. 2. Judgment with respect to each risk of bias item is presented as a percentage for all of the included studies, as shown in Fig. 3.

\section{The pooled results of meta-analysis}

The pooled results indicated that there was no significant difference between the two groups in terms of the lower limb coronal alignment $(\mathrm{RR}=1.20$, 95\% CI $0.28 \sim 5.21$, n.s. Fig. 4a). For coronal alignment of femoral component, no significant difference was noted between the two groups ( $R R=0.65,95 \% C I 0.19 \sim 2.22$, n.s. Fig. 4b). No significant difference was noted between the two groups in the sagittal alignment of femoral component ( $\mathrm{RR}=0.73$, 95\%CI $0.38 \sim 1.41$, n.s. Fig. $4 \mathrm{c}$ ). The blood loss was less in the EM group compared with the IM group $(\mathrm{MD}=-120.34,95 \% \mathrm{CI}-210.08 \sim-30.59$, $\mathrm{P}=0.009$, Fig. 5a). There was no significant difference between the two groups in terms of the operation time $(\mathrm{MD}=1.41,95 \% \mathrm{CI}-1.82 \sim 4.64$, n.s. Fig. $5 \mathrm{~b})$.

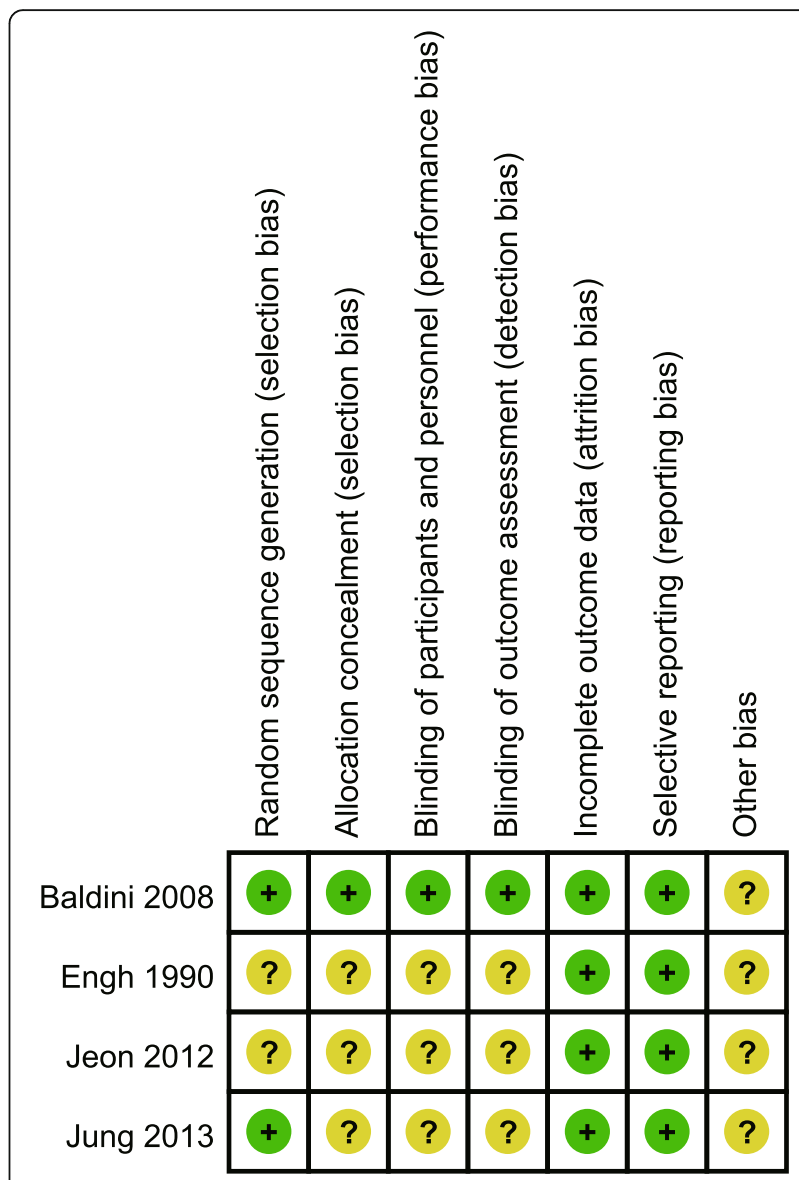

Fig. 2 Methodological quality of included studies. This risk of bias tool incorporates assessment of randomization (sequence generation and allocation concealment), blinding (participants, personnel and outcome assessors), completeness of outcome data, selection of outcomes reported, and other sources of bias. The items were scored with "yes", "no", or "unsure"

\section{Discussion}

Our meta-analysis compared the radiographic outcomes between the EM and the IM femoral guiding technique in patients undergoing TKA. No significant differences were found between the two groups in terms of the lower limb coronal alignment, the coronal alignment of

Table 1 Characteristics of the included studies

\begin{tabular}{|c|c|c|c|c|c|c|}
\hline Author & Country & $\begin{array}{l}\text { Patients } \\
(\text { EM)/(IM) }\end{array}$ & $\begin{array}{l}\text { Age } \\
(E M) /(I M)\end{array}$ & $\begin{array}{l}\text { Gender } \\
(\mathrm{EM}) /(\mathrm{IM})\end{array}$ & Total knee system & EM system \\
\hline Jung 2013 [9] & South Korea & $56 / 50$ & $70.4 / 68.5$ & $\begin{array}{l}\text { Female:male } \\
6: 1 / 5.3: 1\end{array}$ & PS prosthesis (Stryker) & $\begin{array}{l}\text { Mechanical axis marker with } \\
\text { IFD measurement }\end{array}$ \\
\hline Jeon 2012 [28] & South Korea & $40 / 40$ & $70.1 / 69.2$ & Female & PS prosthesis (Stryker) & Markers attached to skin \\
\hline Baldini 2008 [8] & Italy & $50 / 50$ & $71 / 70$ & $\begin{array}{l}\text { Female:male } \\
2: 1 / 1.7: 1\end{array}$ & $\begin{array}{l}\text { Posterior stabilized flex fixed-bearing } \\
\text { prosthesis (Zimmer) }\end{array}$ & $\begin{array}{l}\text { An extramedullary device with } \\
\text { preoperative templated data }\end{array}$ \\
\hline Engh 1990 [7] & USA & $32 / 40$ & $69.11(38-88)$ & $\begin{array}{l}\text { Female: } 53 \\
\text { Male: } 19\end{array}$ & Depuy & $\begin{array}{l}\text { HDisc-peg taped to skin for } \\
\text { intraoperative location }\end{array}$ \\
\hline
\end{tabular}




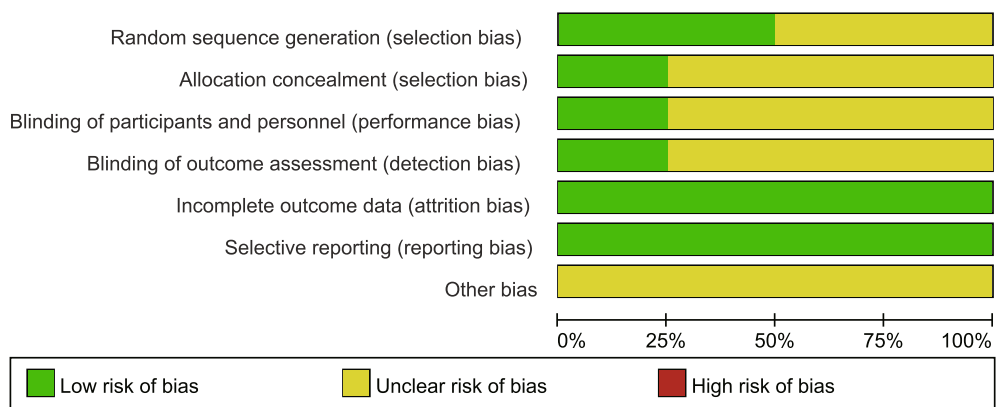

Fig. 3 Risk of bias. Each risk of bias item presented as percentages across all included studies which indicated the proportion of different level risk of bias for each item

femoral component, and the sagittal alignment of femoral component. The EM guide was associated with a less blood loss and exhibited a similar operation time as the IM guide.

Long-term success after TKA is dependent especially on proper intraoperative component positioning [1-4]. Most authors have reported favorable femoral cuts made with the IM guide and it is considered to be the most accurate with a statistically significant increase in the percentage of distal femoral cuts [6,7]. Several studies found $85-96 \%$ of IM femoral cuts to be acceptable compared with $69-86 \%$ of EM cuts. The EM femoral alignment technique had inferior accuracy with approximately $10 \%$ more outliers on the coronal plane compared with the IM technique [9]. Historically, all the authors were using the EM instruments referring only to the anterosuperior iliac spine (ASIS) intraoperatively
$[7,16]$. However, the use of the ASIS to locate the femoral head center (FHC) might not be an accurate method since the FHC was indirectly determined by and dependent on anatomical structures adjacent to the femoral head. Identification of the FHC using the two fingerbreadths medial to the ASIS method was unreliable and a wide variation of inter-ASIS distances was found among patients [17].

Recently, some studies have introduced new techniques to improve the accuracy of the extramedullary alignment guide system. Baldini and Adravanti [8] developed a set of EM instruments calibrated with preoperative templating radiograph measurements of inter-femoral head center distance (IFD), which allowed one to perform distal femoral resection without violating the femoral canal. They reported that the femoral component coronal alignment was within $0^{\circ} \pm 2^{\circ}$ of the
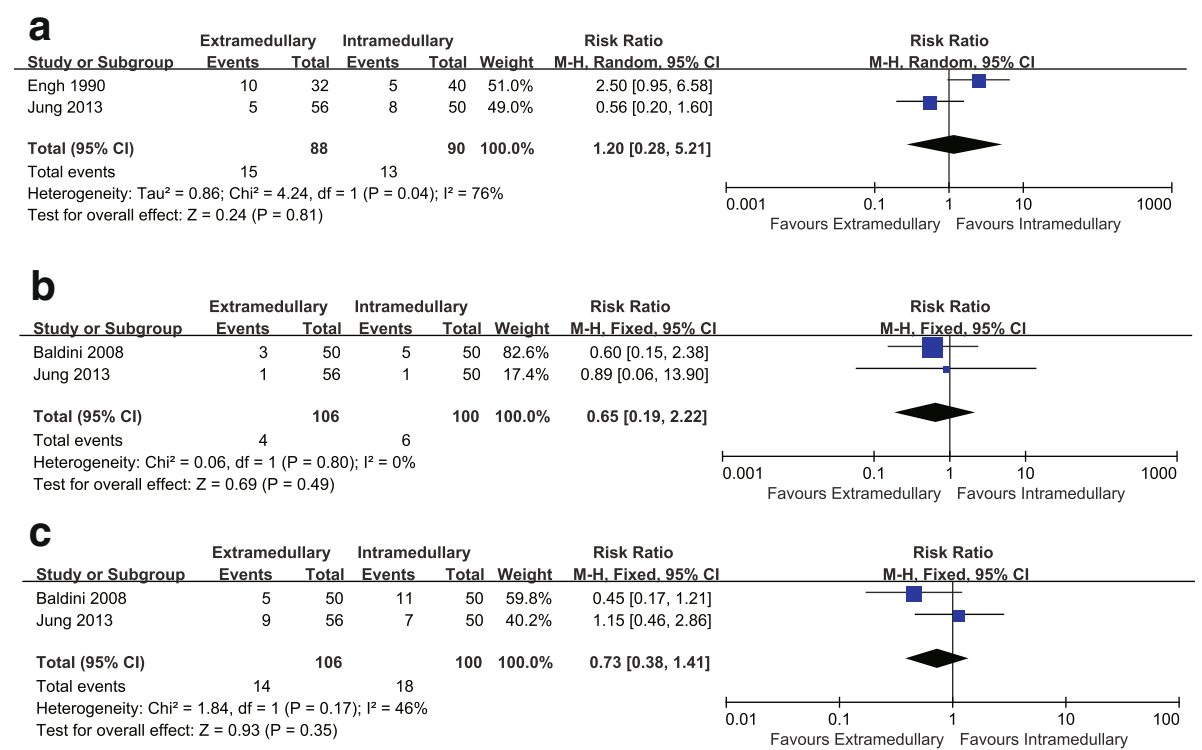

Fig. 4 a Comparison of the lower limb coronal alignment between femoral EM and IM techniques. b Comparison of coronal alignment of femoral component between femoral EM and IM techniques. c Comparison of sagittal alignment of femoral component between femoral EM and IM techniques 


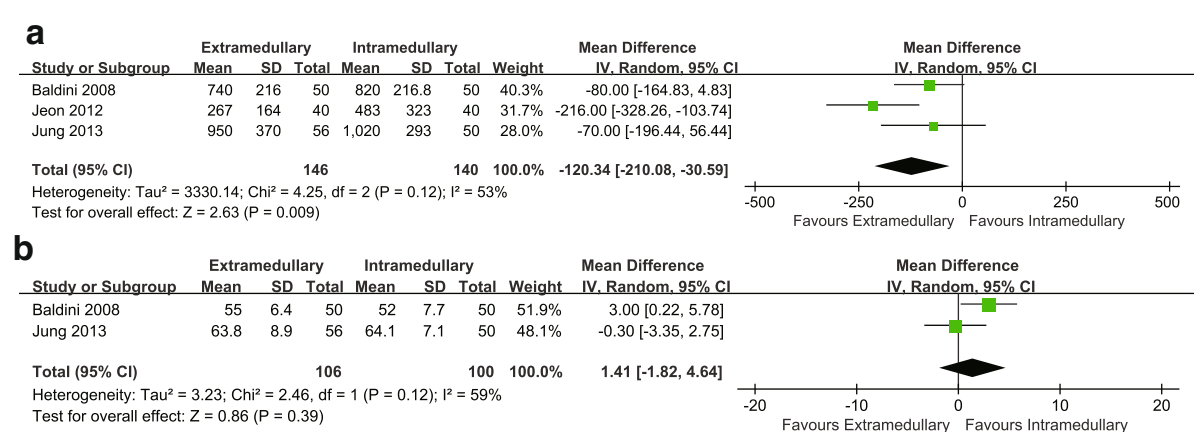

Fig. 5 a Comparison of blood loss between femoral EM and IM techniques. $\mathbf{b}$ Comparison of operative time between femoral EM and IM techniques

mechanical axis in $84 \%$ of the IM group and $86 \%$ of the EM group. Matsumoto et al. [18] reported that the femoral component coronal alignment was within $0^{\circ} \pm 3^{\circ}$ of the mechanical axis in $98 \%$ of patients by using a similar EM instrument. Seo et al. [19] using preoperative templating radiograph measurements of IFD showed that outliers $\left( \pm 3^{\circ}\right)$ of the femoral component coronal alignment were observed in $9.4 \%$ of all cases. However, the proximal reference point in the coronal plane was considered to be incorrect when the lower limb was abducted or adducted during surgery. Therefore, Seo et al. [20] reported the extramedullary technique assisted by a mechanical axis marker, which could easily identify the center of femoral head and result in $98.2 \%$ of patients achieving acceptable alignment in the range of $0^{\circ} \pm 3^{\circ}$ in the coronal plane. Jung et al. [9] reported that the femoral component coronal alignment was within $90^{\circ} \pm 5^{\circ}$ in $98.4 \%$ of patients using the mechanical axis marker system. This new EM alignment guide system was accurate since it included proximal and distal coronal axis markers to indicate the IFD, which was independent of leg posture. The use of an intramedullary guide for the femur was associated with increased risks of fat embolism, blood loss, postoperative hypoxia, and intraoperative fractures [11, 14, 15]. In addition, the IM femoral alignment system did not always guarantee accuracy of the component position in the TKA. The femoral bowing was a common phenomenon and could affect axial alignment of TKA when IM alignment systems were used, especially in East Asian populations $[21,22]$. In these cases, the EM femoral alignment system was a useful alternative surgical option to adjust femoral component alignment [9]. In patients with a more bowed femur, malalignment of lower limb may occur in IM technique, while Computer-assisted techniques could improve the accuracy in both sagittal and coronal planes and EM technique could improve the accuracy in coronal planes. Although the computer-assisted TKA enabled more accurate component alignment [23, 24], the 10-year outcomes of computer-assisted TKA are not superior to that of the conventional technique in function, patient satisfaction and implant survivorship [25, 26].

The opening of the medullary canal using intramedullary jigs was postulated to cause significant blood loss during TKA, although most surgeons have closed the femoral canal opening with bone plug. The application of EM technique was associated with minimal invasiveness since the femoral canal was not breached and the blood loss could be reduced by 145-396 mL [8, 14, 27-29]. Computer-assisted TKA and patient-specific instrumentation (PSI) were recently introduced with the aim of improving alignment without violating the femoral canal [30-32]. TKA using PSI did not result in significantly better femoral component alignment in the sagittal and axial planes than TKA using conventional instrumentation [32]. However, the blood loss was significantly reduced by using the PSI system compared with the IM system for femoral cut. While, the computer-assisted TKA enabled more accurate component alignment. However, it did not reduce the hidden blood loss since the blood loss avoided by not opening the canal might be compensated by greater post tourniquet bleeding due to greater tourniquet time [31].

This present meta-analysis has several limitations. First, only four studies were included and the sample size of the included studies was small, which might have affected our results. Second, we could not perform a valid statistical comparison of the functional outcomes between the two groups. Therefore, further high-quality RCTs with longterm follow-up should be designed to assess radiographic outcomes, knee function, and implant survival rate.

\section{Conclusions}

A satisfactory alignment can be obtained with the use of either intramedullary or extramedullary alignment guide system in TKAs. The use of the extramedullary guide results in less blood loss and exhibits a similar operation time as the intramedullary guide. 


\section{Additional file}

Additional file 1: Comparison of blood loss between femoral EM and IM technique $(P=0.009)$. (DOCX $12 \mathrm{~kb})$

\section{Abbreviations}

Cl: Confidence interval; CNKI: China National Knowledge Infrastructure; EM: Extramedullary; IM: Intramedullary; MD: Mean differences; RCTs: Randomized controlled trials; RR: Risk ratio; TKA: Total knee arthroplasty

\section{Acknowledgements}

We thank all authors of the included studies and the authors listed have made substantial contributions to the study HXL conceived the study design.

\section{Funding}

This work was supported by National Natural Science Foundation of China (81501869)

\section{Availability of data and materials}

We state that the data will not be shared since all raw data used are presented in the figures that are included in the article.

\section{Authors' contributions}

HXL conceived the study design. QT, GZ, and HXL performed the study, collected the data, and contributed to the study design. QT and HXL prepared the manuscript. PS and HZX edited the manuscript. All authors read and approved the final manuscript.

\section{Competing interests}

The authors declare that they have no competing interests.

\section{Consent for publication}

Not applicable.

\section{Ethics approval and consent to participate}

All analyses were based on previous published studies; thus, no ethical approval and patient consent are required.

\section{Publisher's Note}

Springer Nature remains neutral with regard to jurisdictional claims in published maps and institutional affiliations.

\section{Author details}

${ }^{1}$ Department of Orthopaedic Surgery, The Second Affiliated Hospital and Yuying Children's Hospital of Wenzhou Medical University, 109, Xueyuanxi road, 325027 Wenzhou, China. ${ }^{2}$ Department of Rehabilitation, The Second Affiliated Hospital and Yuying Children's Hospital of Wenzhou Medical University, 109 Xueyuanxi road, Wenzhou 325027, China.

Received: 18 February 2017 Accepted: 21 May 2017

Published online: 05 June 2017

\section{References}

1. Fang DM, Ritter MA, Davis KE. Coronal alignment in total knee arthroplasty: just how important is it? J Arthroplasty. 2009;24:39-43.

2. Fujimoto E, Sasahige Y, Tomita T, Kashiwagi K, Inoue A, Sawa M, et al. Different femorotibial contact on the weight-bearing: midflexion between normal and various aligned knees after total knee arthroplasty. Knee Surg Sports Traumatol Arthrosc. 2015;23:1720-8.

3. Kim YH, Park JW, Kim JS, Park SD. The relationship between the survival of total knee arthroplasty and postoperative coronal, sagittal and rotational alignment of knee prosthesis. Int Orthop. 2014:38:379-85.

4. Ritter MA, Davis KE, Meding JB, Pierson JL, Berend ME, Malinzak RA. The effect of alignment and BMI on failure of total knee replacement. J Bone Joint Surg Am. 2011;93:1588-96.

5. Brys DA, Lombardi Jr AV, Mallory TH, Vaughn BK. A comparison of intramedullary and extramedullary alignment systems for tibial component placement in total knee arthroplasty. Clin Orthop Relat Res. 1991;263:175-9.
6. Dennis DA, Channer M, Susman MH, Stringer EA. Intramedullary versus extramedullary tibial alignment systems in total knee arthroplasty. J Arthroplasty. 1993;8:43-7.

7. Engh GA, Petersen TL. Comparative experience with intramedullary and extramedullary alignment in total knee arthroplasty. J Arthroplasty. 1990;5:1-8.

8. Baldini A, Adravanti P. Less invasive TKA extramedullary femoral reference without avigation. Clin Orthop Relat Res. 2008;466:2694-700.

9. Jung $\mathrm{WH}$, Chun $\mathrm{CW}$, Lee $\mathrm{JH}, \mathrm{Ha} \mathrm{JH}$, Jeong JH. The accuracy of the extramedullary and intramedullary femoral alignment system in total knee arthroplasty for varus osteoarthritic knee. Knee Surg Sports Traumatol Arthrosc. 2013;21:629-35.

10. Seo JG, Moon YW, Kim YS. A comparison of extramedullary and intramedullary femoral component alignment guide systems in TKA. J Korean Knee Soc. 2006;18:47-54.

11. Laskin RS. Minimally invasive total knee arthroplasty: the results justify its use. Clin Orthop Relat Res. 2005:440:54-9.

12. Liu HC, Kuo FC, Huang CC, Wang JW. Mini-midvastus total knee arthroplasty in patients with severe varus deformity. Orthopedics. 2015;38:112-7.

13. Xu SZ, Lin XJ, Tong X, Wang XW. Minimally invasive midvastus versus standard parapatellar approach in total knee arthroplasty: a meta-analysis of randomized controlled trials. PLoS One. 2014:9:e95311.

14. Kandel L, Vasili C, Kirsh G. Extramedullary femoral alignment instrumentation reduces blood loss after uncemented total knee arthroplasty. J Knee Surg. 2006;19:256-8.

15. Kumar N, Saleh J, Gardiner E, Devadoss VG, Howell FR. Plugging the intramedullary canal of the femur in total knee arthroplasty: reduction in postoperative blood loss. J Arthroplasty. 2000:15:947-9.

16. Ishii Y, Ohmori G, Bechtold JE, Gustilo RB. Extramedullary versus intramedullary alignment guides in total knee arthroplasty. Clin Orthop Relat Res. 1995:318:167-75.

17. Mullaji A, Shetty GM, Kanna R, Sharma A. Variability in the range of interanterior superior iliac spine distance and its correlation with femoral head centre. A prospective computed tomography study of 200 adults. Skeletal Radiol. 2010:39:363-8.

18. Matsumoto K, Mori N, Ogawa H, Akiyama H. Accuracy of novel extramedullary femoral alignment guide system in primary total kneearthroplasty. Arch Orthop Trauma Surg. 2015;135:1743-8

19. Seo JG, Lim JS, Lee HI, Woo KJ. An extramedullary femoral alignment system in total knee arthroplasty using the inter-femoral head center distance. J Korean Orthop Assoc. 2010:45:24293-300.

20. Seo JG, Moon YW, Park SH, Shim JW, Kim SM. An alternative method to create extramedullary references in total knee arthroplasty. Knee Surg Sports Traumatol Arthrosc. 2012;20:1339-48.

21. Kim JM, Hong SH, Kim JM, Lee BS, Kim DE, Kim KA, et al. Femoral shaft bowing in the coronal plane has more significant effect on the coronal alignment of TKA than proximal or distal variations of femoral shape. Knee Surg Sports Traumatol Arthrosc. 2015;23:1936-42.

22. Lasam MP, Lee KJ, Chang CB, Kang YG, Kim TK. Femoral lateral bowing and varus condylar orientation are prevalent and affect axial alignment of TKA in Koreans. Clin Orthop Relat Res. 2013;471:1472-83.

23. Blakeney WG, Khan RJ, Wall SJ. Computer-assisted techniques versus conventional guides for component alignment in total knee arthroplasty: a randomized controlled trial. J Bone Joint Surg Am. 2011;93(15):1377-84.

24. Todesca A, Garro L, Penna M, Bejui-Hugues J.Conventional versus computernavigated TKA: a prospective randomized study. Knee Surg Sports Traumatol Arthrosc. 2016. [Epub ahead of print].

25. Khuangsirikul S, Lekkreusuwan K, Chotanaphuti T. 10-Year patient satisfaction compared between computer-assisted navigation and conventional techniques in minimally invasive surgery total knee arthroplasty. Comput Assist Surg (Abingdon). 2016;21(1):172-5.

26. Ouanezar $H$, Franck $F$, Jacquel $A$, Pibarot $V$, Wegrzyn J. Does computerassisted surgery influence survivorship of cementless total knee arthroplasty in patients with primary osteoarthritis? A 10-year follow-up study. Knee Surg Sports Traumatol Arthrosc. 2016;24(11):3448-56.

27. Dutton AQ, Yeo SJ, Yang KY, Lo NN, Chia KU, Chong HC. Computer-assisted minimally invasive total knee arthroplasty compared with standard total knee arthroplasty. A prospective, randomized study. J Bone Joint Surg Am. 2008:90:2-9.

28. Jeon SH, Kim JH, Lee JM, Seo ES. Efficacy of extramedullary femoral component alignment guide system for blood saving after total knee arthroplasty. Knee Surg Relat Res. 2012;24:99-103. 
29. Kalairajah Y, Simpson D, Cossey AJ, Verrall GM, Spriggins AJ. Blood loss after total knee replacement: effects of computer-assisted surgery. J Bone Joint Surg (Br). 2005;87:1480-2.

30. Conteduca F, lorio R, Mazza D, Ferretti A. Patient-specific instruments in total knee arthroplasty. Int Orthop. 2014;38:259-65.

31. Singla A, Malhotra R, Kumar V, Lekha C, Karthikeyan G, Malik V. A Randomized controlled study to compare the total and hidden blood loss in computer-assisted surgery and conventional surgical technique of total knee replacement. Clin Orthop Surg. 2015;7:211-6.

32. Yan $\mathrm{CH}$, Chiu KY, Ng FY, Chan PK, Fang CX. Comparison between patientspecific instruments and conventional instruments and computer navigation in total knee arthroplasty: a randomized controlled trial. Knee Surg Sports Traumatol Arthrosc. 2015;23:3637-45.

Submit your next manuscript to BioMed Central and we will help you at every step:

- We accept pre-submission inquiries

- Our selector tool helps you to find the most relevant journal

- We provide round the clock customer support

- Convenient online submission

- Thorough peer review

- Inclusion in PubMed and all major indexing services

- Maximum visibility for your research

Submit your manuscript at www.biomedcentral.com/submit
Biomed Central 